\title{
Spinal Cord Editor's Page October 2006
}

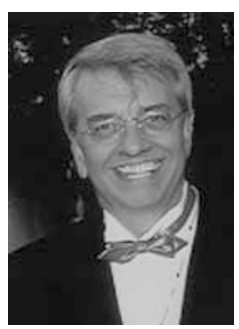

\section{Dear All,}

In this issue of Spinal Cord, a new feature is introduced: the Editor's Page. More changes will gradually occur in the coming months. All of these will be aimed at making the reading and consultation of Spinal Cord even more interesting and enjoyable. Some hints of future developments include: substantial changes will be made to Spinal Cord's Instructions to Authors, to provide clearer submission guidelines; the Editorial Board will be restructured; and plans are in place to reduce our times to print publication. Speed has become very important in the reviewing and publication process - I do want to thank the many reviewers who help us to achieve this.

The October issue gives ample attention to basic animal and clinical research. This month's review looks into the important subject of translating laboratory techniques into practical therapy. Two more animal studies are included: one evaluates the effect of postinjury prednisolone in a spinal cord injury model; the other studies the accuracy of creating an intrinsic locomotor's outcome by precise dorsal spinal cord transsection.

The three clinical articles have direct relevance for practice: the first article analyses the effectiveness of bronchial clearance; the second investigates the use of thermal perception determination to assess the level of spinal lesions; and the third develops and validates a score for neurogenic bowel dysfunction.

Finally, two case reports describe rare clinical cases.

We do hope that you will help us in continuously improving our journal. We, at the Editorial Office, look forward to receiving your suggestions and comments.

Spinal Cord (2006) 44, 583. doi:10.1038/sj.sc.3101980

J-J Wyndaele

Editor-in-Chief

E-mail: Spinalcord@uza.be 